\title{
A Study on Estimating the Next Failure Time of Compressor Equipment in an Offshore Plant
}

\author{
SangJe Cho, ${ }^{1}$ Jong-Ho Shin, ${ }^{2}$ Hong-Bae Jun, ${ }^{3}$ Ho-Jin Hwang, ${ }^{4}$ \\ Chunghun $\mathrm{Ha}^{3}{ }^{3}$ and Jinsang Hwang ${ }^{5}$ \\ ${ }^{1}$ Department of Mechanical Engineering, EPFL (SCI-STI-DK), Lausanne, Switzerland \\ ${ }^{2}$ Department of Industrial Engineering, Chosun University, Kwangju, Republic of Korea \\ ${ }^{3}$ Department of Industrial Engineering, Hongik University, Seoul, Republic of Korea \\ ${ }^{4}$ Korea Research Institute of Ships and Ocean Engineering, Daejeon, Republic of Korea \\ ${ }^{5}$ PartDB Co., Daejeon, Republic of Korea
}

Correspondence should be addressed to Hong-Bae Jun; hongbae@gmail.com

Received 23 June 2016; Accepted 3 October 2016

Academic Editor: Inmaculada T. Castro

Copyright (C) 2016 SangJe Cho et al. This is an open access article distributed under the Creative Commons Attribution License, which permits unrestricted use, distribution, and reproduction in any medium, provided the original work is properly cited.

\begin{abstract}
The offshore plant equipment usually has a long life cycle. During its O\&M (Operation and Maintenance) phase, since the accidental occurrence of offshore plant equipment causes catastrophic damage, it is necessary to make more efforts for managing critical offshore equipment. Nowadays, due to the emerging ICTs (Information Communication Technologies), it is possible to send health monitoring information to administrator of an offshore plant, which leads to much concern on CBM (ConditionBased Maintenance). This study introduces three approaches for predicting the next failure time of offshore plant equipment (gas compressor) with case studies, which are based on finite state continuous time Markov model, linear regression method, and their hybrid model.
\end{abstract}

\section{Introduction}

In general, maintenance is defined as all technical and managerial actions taken during usage period to maintain or restore the required functionality of an asset or equipment. There have been various classifications of maintenance policies. Simply, maintenance policies can be divided into breakdown maintenance (corrective maintenance), preventive maintenance, and CBM (Condition-Based Maintenance). Unlike breakdown maintenance and preventive maintenance, the CBM focuses on not only fault detection and diagnostics of equipment but also degradation monitoring and failure prediction. Generally, CBM can be treated as a method used to reduce the uncertainty of maintenance activities and is carried out according to the requirements indicated by equipment condition [1]. Thus, the CBM enables us to identify and solve problems in advance before equipment damage occurs. In industry systems, any equipment damage can lead to serious results. Since a critical failure or degradation of the equipment during its operation can seriously damage the belief of customers on the equipment reliability, the maintenance enhancement for preventing this kind of failure or degradation in advance has precedence over any other things in a company. Since oil and gas industries are particularly capital-intensive, careful management on equipment is very important. In this respect, the CBM is a very attractive method for oil and gas industries. CBM is currently being utilized in the petrochemical industry, with condition monitoring of both on and offshore oil and gas wells [2].

Until now it has been difficult to achieve the effectiveness of maintenance operations because there is no information visibility during equipment's usage period. However, recently, with emerging technologies such as various sensors, SCADA (Supervisory Control And Data Acquisition) and PEID (Product Embedded Information Devices) are expected to be rapidly used for gathering and monitoring the status data of equipment during equipment usage period. Advancements in information communication technology have added accelerated growth in the CBM technology area 
by enabling network bandwidth, data collection and retrieval, data analysis, and decision support capabilities for large data sets of time series data [3]. Under the new environment, we can gather the equipment status data related to usage conditions, failure, maintenance or service events, and so on. These data sets enable us to diagnose the degradation status of the equipment in a more exact way. Therefore, using this information gives us new challenging issues for improving the efficiency of equipment maintenance operations. We can diagnose equipment status, predict equipment abnormality, and execute proactive maintenance, that is, doing CBM.

Although there have been some relevant research works so far, the CBM is still challenging area. Current approaches have the limitation in detailed methods or validated predictive models, in particular, in the offshore plant domain. This study deals with the approaches that can predict the next failure time of offshore plant equipment (gas compressor) used in LNG FPSO (Liquefied Natural Gas Floating Production Storage and Offloading vessel).

An LNG FPSO is an offshore plant of delivering liquefied gas from a gas field to customers. Recently, the demand for LNG FPSO is highly increasing and the demand for LNG FPSO projects will grow along with the increased demand for natural gas [4]. The O\&M phase of LNG FPSO requires heavy charges and more efforts to optimize the cost and to reduce the risks than the construction phase because of its long life cycle. Nowadays due to the fact that an accident of LNG FPSO in operation causes catastrophic damage, many studies have focused on a maintenance system. In this vein, this study focuses on the prognostic approach for the gas compressor equipment in LNG FPSO, which is one of the main results for the Korean government supported project that is being currently developed since 2013 with the objective of implementing the predictive maintenance system for LNG FPSOs. The objective of this study is to develop the algorithm for estimating the next failure time of a gas compressor based on gathered vibration sensing and failure data. To this end, in this study, finite state Markov model based approach, linear regression model based approach, and their hybrid model have been introduced. To evaluate the proposed approaches, the case study and computational experiments for compressor equipment have been carried out.

The rest of this study is organized as follows. First, relevant previous studies are reviewed and their limitations are discussed in Section 2. Then, Section 3 describes the equipment for CBM focused on this study and two approaches for estimating the next failure time of a compressor, and Section 4 introduces relevant case studies and computational experiments. In Section 5, the hybrid approach combining two approaches is proposed with computational experiment. Finally this study is concluded with further research issues in Section 6.

\section{Literature Review}

There are several maintenance policies: corrective maintenance, preventive maintenance, opportunistic maintenance, Condition-Based Maintenance, and predictive maintenance.
Corrective maintenance is the unplanned maintenance. However, preventive maintenance (such as constant interval maintenance, age based maintenance, and imperfect maintenance) and predictive maintenance (such as RCM (Reliability Centered Maintenance) and CBM) are the types of planned maintenance. For more details, please refer to Bevilacqua and Braglia [5] or Kothamasu et al. [6]. Among various maintenance policies, this study focuses on the CBM.

The term, CBM, is often used with other terms such as PdM (Predictive Maintenance), PHM (Prognostic and Health Management), and on-condition maintenance which comes from the US Department of Defense and Department of Energy. In this study, we define CBM as a maintenance policy which does maintenance action before equipment failures happen, by assessing equipment condition including operating environments and predicting the risk of equipment failures in a real-time way, based on gathered data. The benefits of a successful CBM strategy are expected to include less regular maintenance, the reduction of unscheduled maintenance, and improved supply chain management [7].

Until so far, there have been several research works about CBM. For example, Lee [8] introduced the fundamental technologies for remote maintenance (called teleservice engineering) and CBM: machine performance assessment and remote diagnosis. Lee [9] introduced a new methodology of CBM, called machinery dynamics and data fusion through remote machinery monitoring. He presented an example of a remote wireless application currently in use for monitoring machinery in industrial plants. Dieulle et al. [10] dealt with the problem related to CBM policy for a single-unit deteriorating system and proposed the approach to determine the optimal inspection schedule and replacement threshold with renewal processes theory. Furthermore, Grall et al. [11] dealt with a condition-based inspection/replacement problem for a stochastically and continuously deteriorating single-unit system. With regenerative and semiregenerative processes theory, they tried to find two maintenance decision variables: preventive replacement threshold and inspection schedule, with the objective of minimizing the long run expected maintenance cost per unit time. In addition, Lin and Tseng [12] combined traditional reliability modelling methods with vibration-based monitoring techniques and artificial neural network technologies in an integrated system to determine the health status of machinery, namely, CMAC-PEM (Cerebellar Model Articulation Controller neural network-based machine Performance Estimation Model). They developed a WPHM (Weibull Proportional Hazards Model) and carried out a bearing deterioration experiment to test both the CMAC-PEM and the WPHM. Moore and Starr [13] have reviewed the methods and functionality of criticality assessments in condition-based monitoring and proposed the CBC (Cost Based Criticality) algorithm to rank all the alarms arising from condition monitoring, which could allow optimized prioritization of maintenance activities. Wu et al. [14] proposed an intelligent decision support system for the optimal CBM policy. They developed a neural network model that uses bearing vibration information to predict the life percentage of a machine and the remaining life of the 
machine. They also computed the optimal replacement strategies by proposing a cost matrix method and suggested an optimal replacement time for assisting maintenance decisionmaking. In addition, in their other work [15], they proposed a prognostic method for machine degradation tracking using ARIMA (AutoRegressive Integrated Moving Average) time series model in order to predict the pending failures and the RUL (Remaining Useful Life) of machines. They developed a forecasting strategy and an automatic prediction algorithm of ARIMA models and carried out the analysis on the vibration severity data collected from rotating machines. They compared the performance of the proposed approach with the basic Box-Jenkins ARIMA method. Recently, Hashemian and Bean [16] discussed the limitations of time-based equipment maintenance methods and the advantages of predictive or online maintenance techniques in identifying the onset of equipment failure. Gruber et al. [17] suggested a CBM framework that is based on system simulations and a targeted Bayesian network model. Simulations are used for exploring various CBM policies under different scenarios and the Bayesian network is used for failure prediction based on simulation data. The proposed framework has been applied to a freight rail fleet case. In addition, Lee et al. [18] carried out a comprehensive review of the PHM field. They introduced a systematic PHM design methodology, 5S methodology, for converting data to prognostics information. They also presented a systematic methodology for conducting PHM as applied to machinery maintenance.

In particular, some research works focused on Markovbased model for CBM. For example, Bunks et al. [19] introduced an application of HMMs (Hidden Markov Models) to CBM for the helicopter gearbox case and presented examples of torque level, defect level, and defect-type classification. They concluded that HMMs have a strong potential for constructing practical and robust algorithms for CBM. Furthermore, Ambani et al. [20] developed a continuous time Markov chain degradation model and a cost model to quantify the effects of maintenance on a multiple machine system. To evaluate the effectiveness of the proposed methods, they introduced a case study of an automotive assembly line. In their model, a Markov-based degradation model is used to represent discrete state degradation process, under the assumption that the future degradation state of the machine depends only on the current degradation state and not on the history of the degradation states. Si et al. [21] mentioned that Markovian-based models have been widely applied to RUL estimation and to maintenance decision-making support. They said that the main reason for Markovian-based model is that the plant operation condition can be divided into several meaningful states, such as Good, OK, and Minor defects only, Maintenance required, and Unserviceable, so that the state definition is closer to what is used in industry than other stochastic models and therefore is easy to understand.

On the other hand, some previous works have focused on the maintenance of offshore plant. For example, Wang and Majid [22] carried out a case study of the analysis of reliability and maintenance data on five gas turbines and of the modelling for determining the appropriate preventive maintenance/inspection intervals of offshore oil platform plant. Arthur and Dunn [23] introduced an application of an optimized CBM approach to large reciprocating compressors on an offshore installation. Caselitz and Giebhardt [24] introduced results of work in the field of condition monitoring and fault prediction in offshore wind energy converters. Their work included not only development hardware and software solution but also prototype tests and integration of fault prediction and maintenance and repair scheduling techniques. Furthermore, Dey et al. [25] developed a risk based maintenance model using a combined multiple-criteria decisionmaking and weight method for offshore oil and gas pipelines. Eleye-Datubo et al. [26] and Eleye-Datubo et al. [27] applied Bayesian network methods for examining the system safety of FPSOs. Migueláñez and Lane [28] presented the recovery system of offshore wind turbines. The recovery system takes a broad view of events and sensor values across the complete turbine system and subsystems. In addition, Hussin et al. [29] dealt with a systematic methodology for analyzing the maintenance data of gas compression train system on an offshore platform to gain insight about the system reliability performance and identify the critical factors influencing the performance. Telford et al. [2] explored the existing literature on the development and applications of CBM in the oil and gas industry. de Andrade Melani et al. [30] proposed a method for risk analysis of LNG carriers operations based on Bayesian network method. Recently, Griffith et al. [7] addressed initial development and integration of SHPM (Structural Health and Prognostics Management) system into the O\&M process for offshore wind power plants. They developed a multiscale simulation-based methodology to investigate the sensitivity (or effects) of damage of blades. Cho et al. [31] proposed a linear regression-based approach for estimating the remaining life time of compressor. Cho et al. [32] reviewed previous studies associated with CBM of offshore plants and introduced case studies of prognosis system development predicting performance and failures of offshore plant equipment such as compressor and pump tower.

Although not a few previous research works dealt with various CBM issues, little attention has been paid to the research that deals with offshore plant equipment and has the limitation in estimating the next failure time (remaining life time from the current time) based on gathered sensor data. Estimating the next failure time with sensor data is still the undeveloped area in an offshore plant equipment. To cope with the limitations, this study proposes three approaches to estimate the next failure time of offshore equipment based on finite state continuous time Markov model, linear regression model, and their hybrid model.

\section{Target Equipment and Proposed Approaches}

LNG FPSOs are used when an oil platform is in a remote or deepwater location where seabed pipelines are not cost effective [33]. Nowadays due to the fact that an accident of LNG FPSO in operation causes catastrophic damage, many studies dealt with the improvement of operating a maintenance 


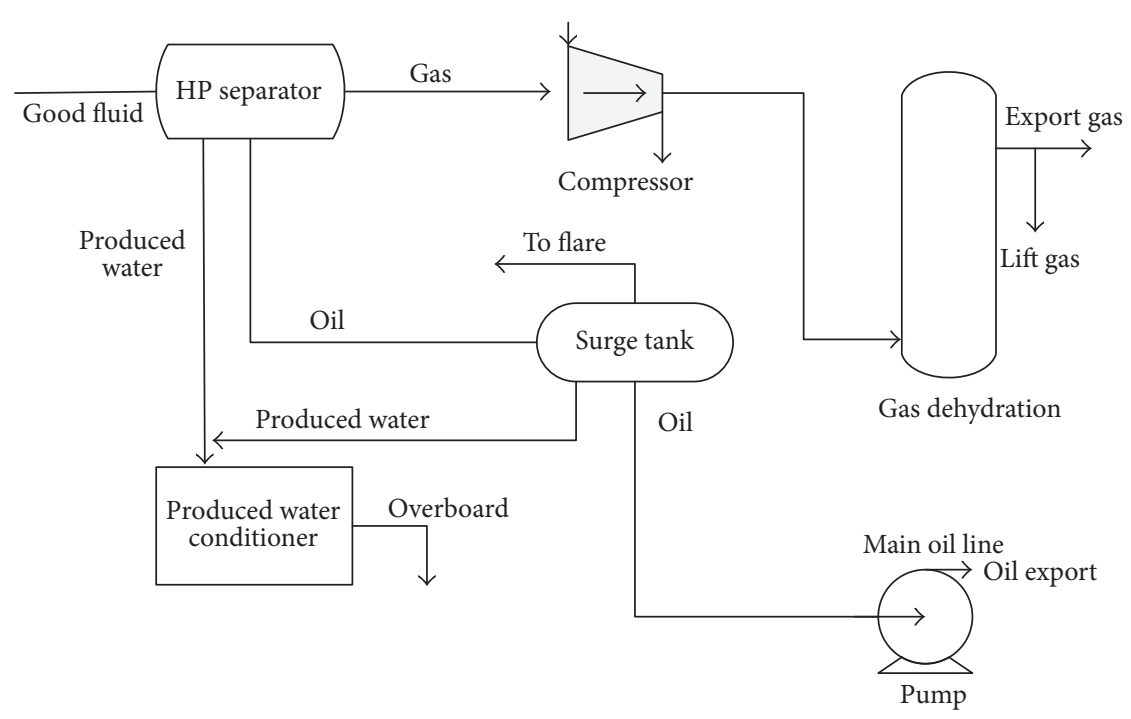

FIGURE 1: Process flow diagram of water, oil, and gas separation in LNG FPSO.

system for LNG FPSO. The LNG FPSO is composed of lots of facilities and equipment. Depending on location, they can be classified into top-side, hull-side, and subsea-side. Among them, this case study focuses on a gas compressor in the topside of LNG FPSO. Figure 1 shows the process flow diagram of water, oil, and gas separation in LNG FPSO. The compressor is used in liquefaction processes of offshore plants. It is important equipment in not only offshore but also onshore plants. It is a mechanical device to increase the pressure of gas and to reduce its volume. It spends most of the energy in offshore plants. Offshore gas compressors are used for various tasks including reservoir management, production enhancement, and the transmission of gas. Any unexpected or prolonged downtime of these units has a large impact on plant availability, as a loss of compression capability drastically affects the oil and gas production of the asset [23]. There are several kinds of compressors. Among them, this study deals with the centrifugal compressor. According to Hussin et al. [29], the number of failures of centrifugal compressor used in an offshore plant is about 26 during six years. For this reason, it preferentially needs to do the development of a prognosis system for the compressor.

According to OREDA [34], frequently observable failure modes of compressor are low output, overheating, spurious stop, external leakage, and so on. The failure mode is usually generated from some failure causes. Compressor failure causes (frequency) are shown as follows:

Rotation/shaft (22\%); instrumentation (21\%); radial bearing (13\%)

Blade/impeller (8\%); thrust bearing (6\%); compressor seal $(6 \%)$

Motor winding (3\%); diaphragm (1\%); and so forth $(20 \%)$

Hence, in this study, we focus on one main cause, rotation/shaft for CBM.
3.1. Vibration Analysis: Compressor. There are some kinds of parameters to monitor the status of the compressor. This study deals with a vibration parameter, because it is widely used in detecting the status of rotating equipment. Generally speaking, vibration is the value with time of the magnitude of a quantity that is descriptive of the motion or position of a mechanical system. Because most normal plant equipment is mechanical, vibration monitoring provides the best tool for routine monitoring and identification of incipient problems [35]. The increasing amplitude of vibration may be an indication of a deteriorating machine condition and the rate of increase is proportional to the degree of damage. Therefore, it is possible to predict the trend of deterioration of a machine by monitoring the amplitudes of its fault related vibration features [36].

Relative shaft vibration and bearing vibration data are usually used to evaluate the status of a compressor of an LNG FPSO. In this study we monitor the status of a gas compressor through relative shaft vibration data. ISO 7919 (international standard for relative shaft vibration of rotating machines) suggests a way for the measurement of a vibration parameter. According to ISO 7919, the max value among two peak-peak values measured by two sensors located in $x$-axis and $y$-axis as 90 degree is expressed as $S_{(\mathrm{p}-\mathrm{p}) \max }=\left[S_{X(\mathrm{p}-\mathrm{p})}, S_{Y(\mathrm{p}-\mathrm{p})}\right]_{\max }$.

A common practice in industry is to set up various warning levels instead of maintenance stages. The warning levels can be classified as alert, high alert, alarm, serious alarm, and breakdown. General guidelines for setting up warning levels for different types of machines are recommended by various national and international committees [36]. ISO 7919 recommends four vibration limits: limit of start-up performance $(A)$, limit of good vibration performance $(B)$, limit for warning alarm $(C)$, and limit for trip $(D)$. The values of limits are calculated as follows: $A / B(4800 / \sqrt{\pi}), B / C$ $(9000 / \sqrt{\pi})$, and $C / D(13200 / \sqrt{\pi})$ where $\pi$ denotes the RPM (Revolution Per Minute) of a compressor. 


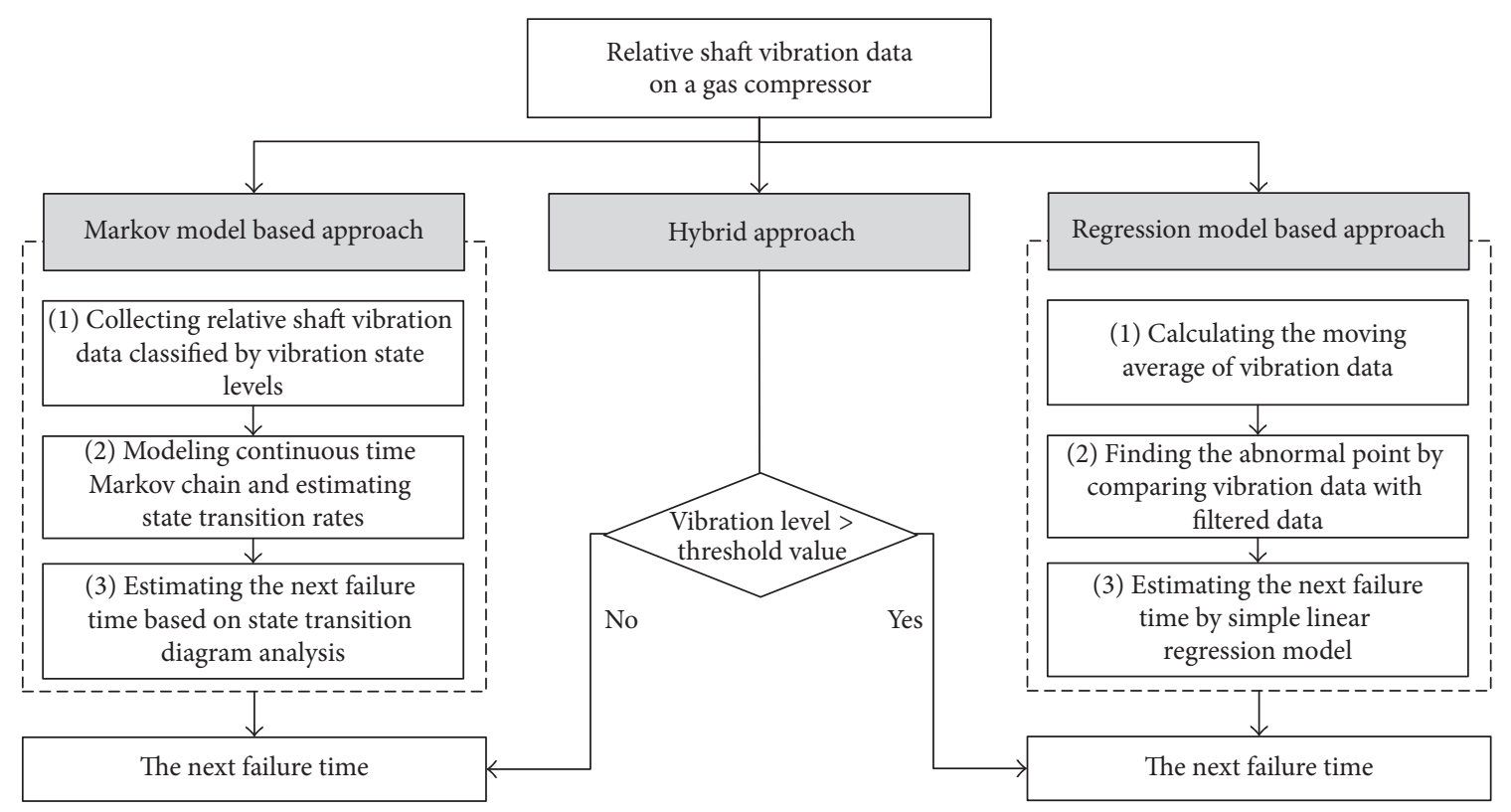

FIGURE 2: Overview of proposed approaches.

In this study, we propose three approaches for estimating the next failure time of a compressor: (1) Markov model based approach, (2) regression model based approach, and (3) hybrid approach combining (1) and (2). In the Markov model based approach, we divide the vibration value into some levels and stochastically predict the next failure time based on the finite state continuous time Markov model theory. In the regression model based approach, when the vibration data evidently differs from the moving average filter and shows increasing trends, we predict the next failure time using simple linear regression model. The hybrid approach takes the advantage of two approaches. Depending on the vibration level, one of two approaches is applied to estimate the next failure time. Figure 2 depicts the detailed process flow of these approaches.

3.2. Markov Model Based Approach. In order to apply the CBM policy of an LNG FPSO compressor, among various prognostics methods such as wavelets, artificial neural networks, and Bayesian network, in this study, we use the finite state continuous time Markov model since it has the benefit in the amount of data required for analysis, compared to other methods. Furthermore, it allows an exact computation of system reliability.

In this study, it is assumed that LNG FPSO operation system records $S_{(\mathrm{p}-\mathrm{p}) \max }$ and its timestamp data whenever the level of relative shaft vibration exceeds the predefined limit (e.g., warning level and trip level). Furthermore, the amplitude of vibration signal of the compressor will remain the limit of tolerance range unless it has abnormal symptoms for faults or failures. In addition, we assume that the vibration state evolves continuously over time and state transition rate does not depend on time based on the interview result with compressor engineers.
To maintain brevity and consistency, this study defines the following notation.

$$
\begin{aligned}
& s: \text { index of compressor state } \\
& \tau_{s, s^{\prime}}: \text { the number of changes from } s \text { to } s^{\prime} \\
& T_{s, s^{\prime}}: \text { transition rate from } s \text { to } s^{\prime} \\
& t_{s, s^{\prime}}^{n}: \text { the time interval of the } n \text {th status transition from } \\
& \text { status } s \text { to status } s^{\prime} \\
& L_{s, s^{\prime}}: \text { the expected time to reach the state } s^{\prime} \text { from the } \\
& \text { state } s \\
& E_{s, s^{\prime}}: \text { the expected time from status } s \text { to the very next } \\
& \text { state } s^{\prime} \\
& P_{s}: \text { the probability to transit from state } s \text { to state } s+1 \\
& P_{s}^{j}: \text { the probability of the jth return to the state } s \text { after } \\
& \text { going through state } s-1 \\
& \pi \text { : the RPM (Revolution Per Minute) of a compressor } \\
& \lambda: \text { the deteriorating transition rate } \\
& \mu \text { : the recovery transition rate }
\end{aligned}
$$

The detailed procedures of the proposed approach are as follows.

Step 1 (collecting relative shaft vibration data classified by vibration state levels). We assumed that LNG FPSO operation system records $S_{(\mathrm{p}-\mathrm{p}) \max }$ and its timestamp data whenever the level of relative shaft vibration exceeds the predefined limit. Regarding the predefined limit, based on ISO 7919, we set the vibration levels into good vibration level and alarm level. And we divide the alarm level into low, middle, and high levels, in detail, because it is more important to predict the next failure time in an alarm level than in a good vibration 


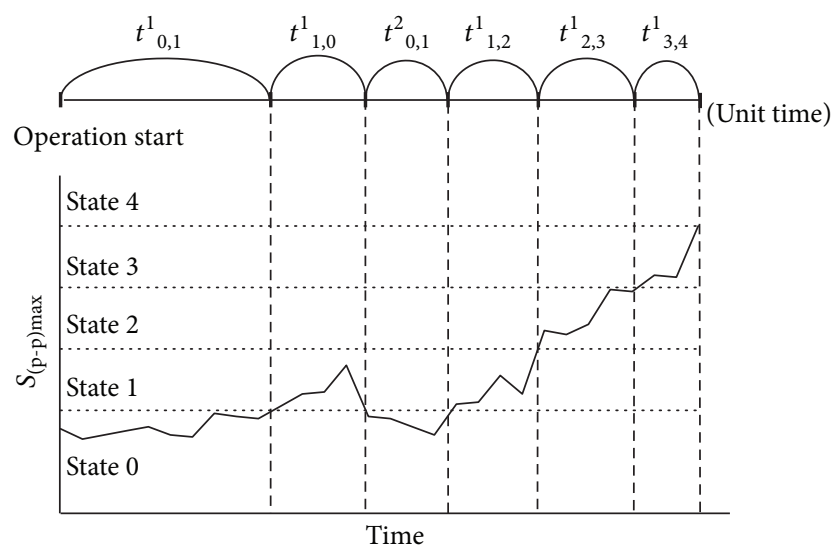

- - - Event occurrence

Figure 3: Example of vibration state transition plot in Markov model based approach.

performance level. Thus, whenever the vibration data exceeds over the predefined levels, the state values (denoted as $s$ ) for representing each level and its timestamp data are recorded. The value of state $s$ has the following meaning:

$$
\begin{aligned}
& s=0: \text { a good vibration level under } 9000 / \sqrt{\pi} \\
& s=1 \text { : a low alarm level under } 10400 / \sqrt{\pi} \\
& s=2: \text { a middle alarm level under } 11800 / \sqrt{\pi} \\
& s=3: \text { a high alarm level under } 13200 / \sqrt{\pi} \\
& s=4: \text { a trip level above } 13200 / \sqrt{\pi}
\end{aligned}
$$

Figure 3 depicts an example of transition plot representing the state values of vibration data changed over time.

Step 2 (estimating status transition rates). From the interview with compressor maintenance engineers, we identified that the failures of the compressor shaft are not much related to the deterioration process and occur randomly. Hence, in this study, we assume that the time when status transition occurs follows the homogeneous Poisson process having the state transition rate as follows:

$$
T_{s, s^{\prime}}=\frac{\tau_{s, s^{\prime}}}{\sum_{k=1}^{\tau_{s, s^{\prime}}} t_{s, s^{\prime}}^{k}} .
$$

Then, since the state transition rate is constant and does not depend on the time, we assume that it has the Markovian property.

Step 3 (estimating the next failure time). Then, $L_{0, s}$ could be estimated by the following formula:

$$
\begin{aligned}
& L_{0, s} \\
& \qquad \sum_{j=0}^{\infty}\left[P_{s-1}^{j} \cdot P r_{s-1} \cdot\left\{j \cdot\left(L_{s-2, s-1}+E_{s-1, s-2}\right)+E_{s-1, s}\right\}\right] \\
& \quad+L_{0, s-1},
\end{aligned}
$$

where $L_{0,0}=0$ and $L_{0,1}=1 / \lambda_{0}, s^{\prime}=2,3,4$.
Let $j$ be the number of first passage from $s^{\prime}-1$ to $s^{\prime}-2$. The first term of formula (2) indicates the expected time until arriving at state $s$ after the $j$ th return to state $s-1$, calculated by multiplying the duration time; that is, $\left(j \cdot\left(L_{s-2, s-1}+E_{s-1, s-2}\right)+\right.$ $\left.E_{s-1, s}\right)$ and its probability $\left(P_{s-1}^{j} \cdot P r_{s-1}\right)$. The second term (i.e., $L_{0, s-1}$ ) of formula (2) denotes the expected time from state 0 to state $s-1$.

Let $\lambda_{s}=T_{s, s+1}, 0 \leq \lambda_{s} \leq 1$, for $s=0,1,2,3$, which indicates the deteriorating transition rate, and $\mu_{s}=T_{s+1, s}$, $0 \leq \mu_{s} \leq 1$, for $s=0,1,2,3$, which indicates the recovery transition rate, respectively. Then, according to Anderson [37], $E_{s-1, s}=1 / \lambda_{s-1}, E_{s, s-1}=1 / \mu_{s}, P_{s}^{j}=\left(\mu_{s} /\left(\lambda_{s}+\mu_{s}\right)\right)^{j}$, and $\operatorname{Pr}_{s-1}=\left(\lambda_{s} /\left(\lambda_{s}+\mu_{s}\right)\right)$ in the continuous time Markov model. As a result, formula (2) could be unfolded as follows:

$$
\begin{aligned}
& L_{0, s}=\sum_{j=0}^{\infty}\left[P_{s-1}^{j} \cdot P r_{s-1}\right. \\
& \left.\cdot\left\{j \cdot\left(L_{s-2, s-1}+E_{s-1, s-2}\right)+E_{s-1, s}\right\}\right]+L_{0, s-1} \\
& =\sum_{j=0}^{\infty}\left[\left(\frac{\mu_{s-1}}{\lambda_{s-1}+\mu_{s-1}}\right)^{j} \cdot\left(\frac{\lambda_{s-1}}{\lambda_{s-1}+\mu_{s-1}}\right)\right. \\
& \left.\cdot\left\{j \cdot\left(L_{s-2, s-1}+\frac{1}{\mu_{s-1}}\right)+\frac{1}{\lambda_{s-1}}\right\}\right]+L_{0, s-1} \\
& =\sum_{j=0}^{\infty}\left[\left(\frac{\mu_{s-1}}{\lambda_{s-1}+\mu_{s-1}}\right)^{j} \cdot\left(\frac{\lambda_{s-1}}{\lambda_{s-1}+\mu_{s-1}}\right)\right. \\
& \left.\cdot\left\{j \cdot\left(L_{s-2, s-1}+\frac{1}{\mu_{s-1}}\right)\right\}\right]+\sum_{j=0}^{\infty}\left[\left(\frac{\mu_{s-1}}{\lambda_{s-1}+\mu_{s-1}}\right)^{j}\right. \\
& \left.\cdot\left(\frac{\lambda_{s-1}}{\lambda_{s-1}+\mu_{s-1}}\right) \cdot \frac{1}{\lambda_{s-1}}\right]+L_{0, s-1} \\
& =\sum_{j=0}^{\infty}\left[\left(\frac{\mu_{s-1}}{\lambda_{s-1}+\mu_{s-1}}\right)^{j} \cdot\left(\frac{\lambda_{s-1}}{\lambda_{s-1}+\mu_{s-1}}\right)\right. \\
& \left.\cdot\left\{(j+1) \cdot\left(L_{s-2, s-1}+\frac{1}{\mu_{s-1}}\right)\right\}\right] \\
& -\sum_{j=0}^{\infty}\left[\left(\frac{\mu_{s-1}}{\lambda_{s-1}+\mu_{s-1}}\right)^{j} \cdot\left(\frac{\lambda_{s-1}}{\lambda_{s-1}+\mu_{s-1}}\right)\right. \\
& \left.\cdot\left(L_{s-2, s-1}+\frac{1}{\mu_{s-1}}\right)\right]+\sum_{j=0}^{\infty}\left[\left(\frac{\mu_{s-1}}{\lambda_{s-1}+\mu_{s-1}}\right)^{j}\right. \\
& \left.\cdot\left(\frac{\lambda_{s-1}}{\lambda_{s-1}+\mu_{s-1}}\right) \cdot \frac{1}{\lambda_{s-1}}\right]+L_{0, s-1}=\left(\frac{\lambda_{s-1}}{\lambda_{s-1}+\mu_{s-1}}\right) \\
& \cdot\left(L_{s-2, s-1}+\frac{1}{\mu_{s-1}}\right) \cdot \sum_{j=0}^{\infty}\left[\left(\frac{\mu_{s-1}}{\lambda_{s-1}+\mu_{s-1}}\right)^{j} \cdot(j+1)\right] \\
& -\left(L_{s-2, s-1}+\frac{1}{\mu_{s-1}}\right)+\frac{1}{\lambda_{s-1}}+L_{0, s-1} .
\end{aligned}
$$




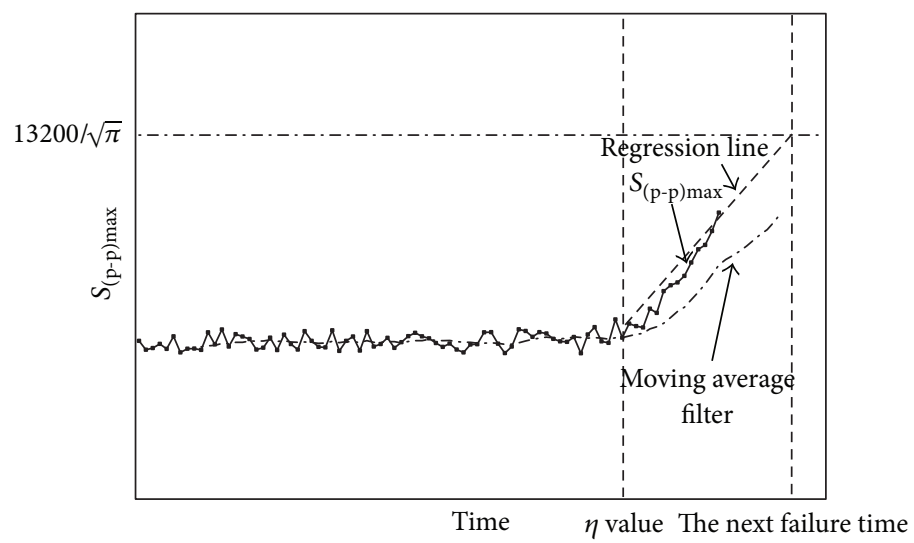

FIGURE 4: Example of the trend plot of vibration data in regression model based approach.

Since

$$
\begin{aligned}
\sum_{j=0}^{\infty}\left[K^{j} \cdot(j+1)\right] & =\frac{d}{d K} \sum_{j=0}^{\infty} K^{j+1}=\frac{d}{d K}\left(\frac{K}{1-K}\right) \\
& =\frac{1}{(1-K)^{2}},
\end{aligned}
$$

where $K=\mu_{s-1} /\left(\lambda_{s-1}+\mu_{s-1}\right)$, the above equation could be expressed as follows.

$$
\begin{aligned}
= & \left(\frac{\lambda_{s-1}}{\lambda_{s-1}+\mu_{s-1}}\right) \cdot\left(L_{s-2, s-1}+\frac{1}{\mu_{s-1}}\right) \\
& \cdot \frac{1}{\left[1-\left(\mu_{s-1} /\left(\lambda_{s-1}+\mu_{s-1}\right)\right)\right]^{2}}-\left(L_{s-2, s-1}+\frac{1}{\mu_{s-1}}\right) \\
& +\frac{1}{\lambda_{s-1}}+L_{0, s-1} \\
= & \frac{\left(\lambda_{s-1} /\left(\lambda_{s-1}+\mu_{s-1}\right)\right)-\left[1-\mu_{s-1} /\left(\lambda_{s-1}+\mu_{s-1}\right)\right]^{2}}{\left[1-\mu_{s-1} /\left(\lambda_{s-1}+\mu_{s-1}\right)\right]^{2}} \\
& \cdot\left(L_{s-2, s-1}+\frac{1}{\mu_{s-1}}\right)+\frac{1}{\lambda_{s-1}}+L_{0, s-1} \\
= & \frac{\left(\lambda_{s-1} \cdot \mu_{s-1}\right) /\left(\lambda_{s-1}+\mu_{s-1}\right)^{2}}{\left[1-\mu_{s-1} /\left(\lambda_{s-1}+\mu_{s-1}\right)\right]^{2}} \cdot\left(L_{s-2, s-1}+\frac{1}{\mu_{s-1}}\right) \\
& +\frac{1}{\lambda_{s-1}}+L_{0, s-1} \\
= & \frac{\mu_{s-1}}{\lambda_{s-1}} \cdot\left(L_{s-2, s-1}+\frac{1}{\lambda_{s-1}}\right)+\frac{1}{\lambda_{s-1}}+L_{0, s-1} \cdot
\end{aligned}
$$

Along formula (5), it is possible to estimate $L_{0,4}$ as follows:

$$
L_{0,4}=\left[\frac{\mu_{3}}{\lambda_{3}} \cdot\left\{L_{2,3}+\frac{1}{\mu_{3}}\right\}\right]+\frac{1}{\lambda_{3}}+L_{0,3} .
$$

Also, we can know that the expected time to reach state $s$ from state $s-1$ is simply calculated as follows.

$$
L_{s-1, s}=L_{0, s}-L_{0, s-1} \text {. }
$$

Along formulae (5) and (7), $L_{0,3}$ and $L_{2,3}$ in formula (6) could be estimated as follows:

$$
\begin{aligned}
& L_{0,3}=\left[\frac{\mu_{2}}{\lambda_{2}} \cdot\left\{L_{1,2}+\frac{1}{\mu_{2}}\right\}\right]+\frac{1}{\lambda_{2}}+L_{0,2}, \\
& L_{2,3}=L_{0,3}-L_{0,2} .
\end{aligned}
$$

$L_{0, s}$ and $L_{s-1, s}$ in a series of calculations like $L_{0,2}$ and $L_{1,2}$ in formula (8) could be estimated using formulae (5) and (7) in the same way.

If the current state is $s$, then $L_{s, 4}$ could be estimated as follows:

$$
L_{s, 4}=L_{0,4}-L_{0, s} .
$$
follows:

Then, finally the next failure time could be estimated as

The next failure time $=$ the current time $+L_{s, 4}$.

3.3. Regression Model Based Approach. In addition to the Markov model based approach, in this study, the regression model based approach for estimating the next failure time of the compressor is proposed. The regression model based approach is based on the $S_{(\mathrm{p}-\mathrm{p}) \max }$ trend plot (please refer to Figure 4). The trend plot is a method to record the variation of the magnitude that is descriptive of motion or positions of the equipment with time. The trend plot helps engineers figure out the status of the equipment at a glance. After the raw data of vibration is recorded in the trend plot, the regression model based approach analyzes $S_{(\mathrm{p}-\mathrm{p}) \max }$ trend plot with moving average filter and abnormal indicator variable (denoted as $v_{i}$ ). According to ISO 7919, we let the limit for trip be a critical limit for the failure of a compressor. Then, it calculates the point where the simple linear regression line intersects with the limit for trip and considers it as the next failure time.

To maintain brevity and consistency, this study defines the following notation.

$k$ : the number of $y_{i}^{\prime}$ s

$m$ : index for calculating $\eta$ 
$n$ : the number of entities used in the moving average filter

$r$ : the number of comparisons with $y_{i}$ and $\bar{y}_{i}$

$v_{i}$ : the 0-1 binary variable that indicates whether $\bar{y}_{i}<$ $y_{i}$ or not (i.e., whether abnormal situation occurs or not)

$x_{i}$ : the time when the $i$ th $S_{(\mathrm{p}-\mathrm{p}) \max }$ value (relative shaft vibration data) is recorded

$y_{i}$ : the $i$ th $S_{(\mathrm{p}-\mathrm{p}) \max }$ value

$\bar{y}_{i}$ : moving average filter of $y_{i}$

$\eta$ : the time point just before the vibration value is continuously over the moving average filter value with $r$ times

$\pi$ : the RPM (Revolution Per Minute) of a gas compressor

The detailed procedure is as follows.

Step 1 (calculating the moving average of vibration data). In this study, in order to predict the next failure time, it is necessary to carefully monitor the trend of vibration data over time points. Since the vibration value itself has the limitation in giving the trend of time series values, the moving average filter is applied to catch the trend of vibration values. If there are $y_{1}, y_{2}, \ldots, y_{k}$, a moving average filter $\bar{y}_{i}$ for $S_{(\mathrm{p}-\mathrm{p}) \max }$ is calculated as follows:

$$
\bar{y}_{i}=\frac{y_{i-n+1}+y_{i-n+2}+\cdots+y_{i}}{n}, \quad n \leq i \leq k .
$$

Step 2 (finding the abnormal time point by comparing vibration data with filtered data). If there is the time point showing abnormal situations continuously compared to previous data, we assume that the failure propagation evolves in a fast way after that point. In this study, to find the abnormal time point, $\eta$ is calculated by formula (13). Here, $\eta$ indicates the time point just before showing the abnormal performance (i.e., $\bar{y}_{i}<y_{i}$ ) continuously in $r$ times.

$$
\eta=\left\{\underset{m}{\arg \min }\left(\prod_{i=m}^{m+r-1} v_{i}\right)\right\}, \quad n \leq m \leq k-r+1,
$$

where

$$
v_{i}=\left\{\begin{array}{ll}
1 & \left(\text { if } \bar{y}_{i}<y_{i}\right) \\
0 & \text { (otherwise) }
\end{array} \quad \text { for } n \leq i \leq k .\right.
$$

Step 3 (estimating the next failure time by a linear regression model). The next failure time could be estimated based on the values of $x_{\eta+1}, x_{\eta+2}, \ldots, x_{k}$ by a linear regression model. If the regression equation is expressed as $y_{i}=\widehat{\beta}_{0}+\widehat{\beta}_{1} \cdot x_{i}$ where $\widehat{\beta}_{0}=\bar{y}-\widehat{\beta}_{1} \cdot \bar{x}$ and $\widehat{\beta}_{1}=\sum_{i=\eta+1}^{k}\left(x_{i}-\bar{x}\right)\left(y_{i}-\bar{y}\right) / \sum_{i=\eta+1}^{k}\left(x_{i}-\bar{x}\right)^{2}$, then the next failure time could be estimated by formula (15):

$$
\text { The next failure time }=\frac{1}{\widehat{\beta}_{1}} \cdot\left(\frac{13200}{\sqrt{\pi}}-\widehat{\beta}_{0}\right) \text {. }
$$

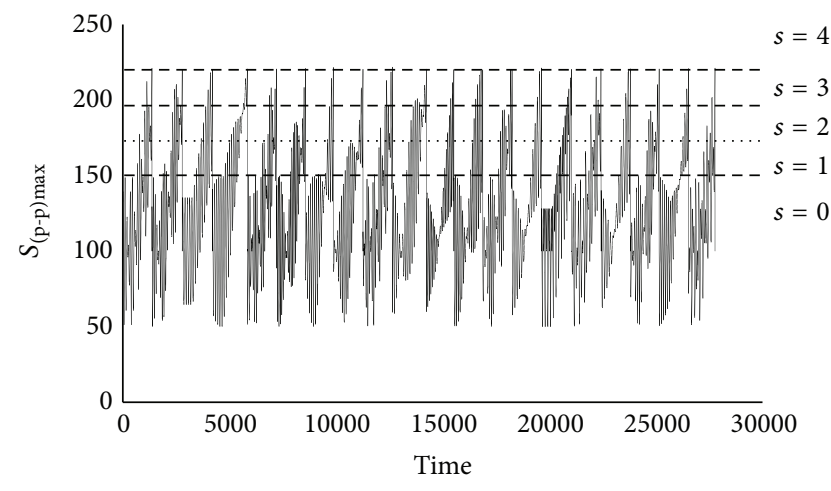

FIGURE 5: Example of vibration data.

\section{Case Study}

For a case study, we used the data on shaft vibration of a gas compressor generated based on the sample vibration data obtained from a compressor manufacturing company in South Korea. The generated data for about three years includes 20 failures and about 564 state transitions, where $\mathrm{RPM}$ is 3600 (i.e., $\pi=3600$ ). The vibration data used in this study is depicted by Figure 5.

4.1. Case Study of Markov Model Based Approach. From the example data of the case study, we obtain the number of changes from $s$ to $s^{\prime}$, that is, $\tau_{s, s^{\prime}}$ as follows: $\tau_{0,1}=135$, $\tau_{1,0}=115, \tau_{1,2}=108, \tau_{2,1}=88, \tau_{2,3}=59, \tau_{3,2}=39$, and $\tau_{3,4}=20$. Here, we describe how to calculate the transition rate, $\lambda_{3}$, that is, $T_{3,4}$. Other calculations for $T_{s, s^{\prime}}$ are omitted for the convenience.

$\lambda_{3}$ could be estimated as follows:

$$
\lambda_{3}=\frac{\tau_{3,4}}{\sum_{k=1}^{\tau_{3,4}} t_{3,4}^{k}}=\frac{20}{15+14+15+\cdots+13}=0.0738
$$

We could obtain the transition rates in the same way as follows: $\lambda_{0}=0.0068, \lambda_{1}=0.0506, \lambda_{2}=0.0569, \mu_{1}=0.0580$, $\mu_{2}=0.0453$, and $\mu_{3}=0.0392$. The state transition diagram with these transition rates is depicted in Figure 6.

Where the current state is the state 0 , the expected time to reach the failure from the state $0\left(L_{0,4}\right)$ could be estimated along formula (6) as follows:

$$
L_{0,4}=\left[\frac{0.0392}{0.0738} \cdot\left\{L_{2,3}+\frac{1}{0.0392}\right\}\right]+\frac{1}{0.0738}+L_{0,3} .
$$

In formula (17), $L_{2,3}$ and $L_{0,3}$ could be estimated in a series of calculations as follows:

$$
\begin{aligned}
& L_{2,3}=L_{0,3}-L_{0,2}, \\
& L_{0,3}=\left[\frac{0.0453}{0.0569} \cdot\left\{L_{1,2}+\frac{1}{0.0453}\right\}\right]+\frac{1}{0.0569}+L_{0,2},
\end{aligned}
$$




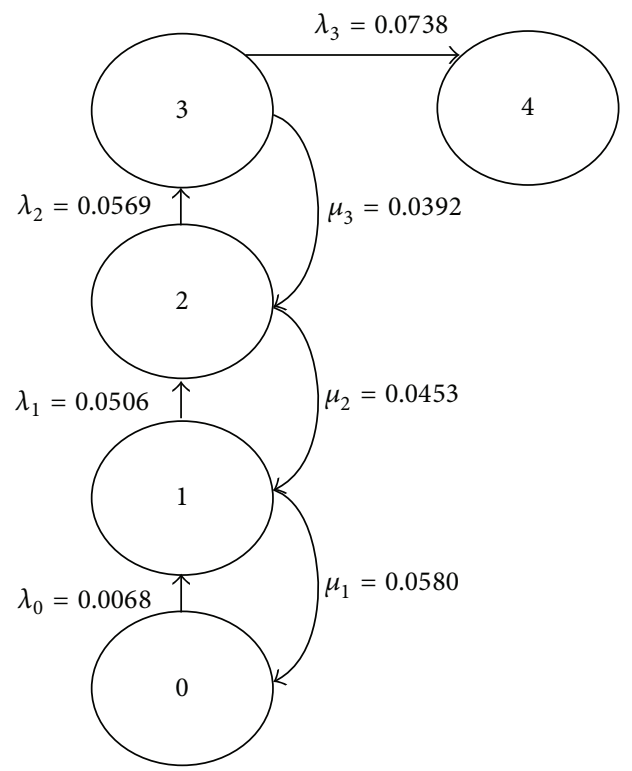

State

(0) Good vibration performance

(1) A warning alarm (low)

(2) A warning alarm (middle)

(3) A warning alarm (high)

(4) A trip

FIGURE 6: Example of state transition diagram.

$$
\begin{aligned}
& L_{0,2}=\left[\frac{0.0580}{0.0506} \cdot\left\{L_{0,1}+\frac{1}{0.0580}\right\}\right]+\frac{1}{0.0506}+L_{0,1}, \\
& L_{0,1}=\frac{1}{0.0068}=147.9778 .
\end{aligned}
$$

Table 1 shows a result of a series of the above formulae. The time point when we estimated the next failure time is 27628 (hours). Since $S_{(\mathrm{p}-\mathrm{p}) \max }$ value is 197.8346 at that time, the vibration state is the state level 3. Thus, the next failure time is as follows: the current time point $+L_{3,4}=27628+134.17=$ 27762.17 (hours). The real failure time is known as 27776 . The residual life time at 27628 time point is only about 134 (hours). Hence, we could see that the estimated next failure time by Markov model based approach is close to the real one.

4.2. Computational Experiments of Markov Model Based Approach. In order to evaluate the performance of the Markov model based approach, we have carried out computational experiments based on the vibration history data (refer to Figure 5). To quantify the degree of the performance of the proposed approach, this study uses the MAPE (Modified Absolute Percentage Error [38]) measure represented as follows:

$$
\text { MAPE }=\left|\frac{R-R^{*}}{\left(R+R^{*}\right) / 2}\right| \cdot 100,
$$

TABLE 1: $L_{s, s^{\prime}}$ calculations.

\begin{tabular}{lc}
\hline$n$ & $t_{3,4}^{n}$ \\
\hline$L_{0,4}$ & 693.3072 \\
$L_{0,3}$ & 559.1403 \\
$L_{0,2}$ & 357.3453 \\
$L_{0,1}$ & 147.9778 \\
$L_{3,4}$ & 134.1669 \\
$L_{2,3}$ & 201.7950 \\
$L_{1,2}$ & 209.3675 \\
\hline
\end{tabular}

TABLE 2: Test results of Markov model based approach.

\begin{tabular}{lccc}
\hline Failure event & $\begin{array}{c}\text { Average } \\
\text { MAPE }^{*}\end{array}$ & $\begin{array}{c}\# \text { obs. less } \\
\text { than } 30 \%^{\dagger}\end{array}$ & $\begin{array}{c}\# \text { obs. less } \\
\text { than } 50 \%^{*}\end{array}$ \\
\hline 1 & 0.308 & 6 & 8 \\
2 & 0.310 & 5 & 8 \\
3 & 0.498 & 2 & 6 \\
4 & 0.407 & 5 & 6 \\
5 & 0.653 & 3 & 4 \\
6 & 0.422 & 5 & 7 \\
7 & 0.377 & 3 & 5 \\
8 & 0.667 & 5 & 5 \\
9 & 0.438 & 6 & 8 \\
10 & 0.644 & 2 & 5 \\
11 & 0.485 & 4 & 5 \\
12 & 0.387 & 5 & 5 \\
13 & 0.258 & 6 & 10 \\
\hline Average & 0.450 & 4.380 & 6.310 \\
\hline
\end{tabular}

${ }^{*}$ The average on 10 MAPEs for each failure event.

${ }^{\dagger}$ Number of observations such that MAPE $<30 \%$ among 10 test examples.

*Number of observations such that MAPE $<50 \%$ among 10 test examples.

where $R^{*}$ indicates residual time to the next failure and $R$ is the estimated residual time to the next failure.

With MAPE, we could avoid the problem of large errors when the residual life time to the next failure is close to zero. To calculate the value of MAPE, in the computational experiment, we use the data after 10000 hours, because Markov model based approach needs enough history data. And then, we randomly chose ten time points for each failure time and estimated the failure time at those times. Table 2 shows that the average MAPE of Markov model based approach is $45.03 \%$. Observation results for the number of solutions less than $30 \%$ or $50 \%$ showed us that a few solutions have high MAPE values so that they seemed to affect the overall performance of the approach. Furthermore, we could find that Markov model based approach has more large MAPEs as the time point becomes close to the real failure time.

4.3. Case Study of Regression Model Based Approach. The case study of regression model based approach has been carried 


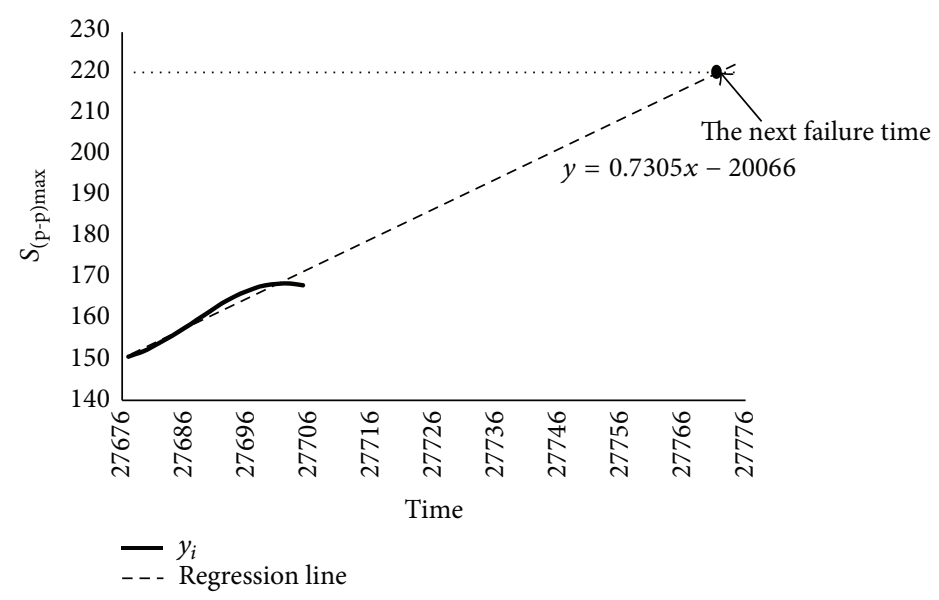

FIGURE 7: Example of regression model based approach.

out based on 100 time point data sets as shown in Table 3. Note that $y_{i}$ is the maximum $S_{(\mathrm{p}-\mathrm{p}) \max }$ value at time $i$ whose unit time is an hour. We set parameters as follows: $n=10$, $r=5$, and $k=27705$. Table 3 shows moving average filter $\bar{y}_{i}$, indicator variable $v_{i}$, and $\prod_{i=m}^{m+r-1} v_{i}$ for the time point $x_{i}$. Along formula (13), $\eta$ is 27676 in this case study.

Figure 7 depicts how to estimate the next failure time. The linear regression model in this case study is calculated as $y=$ $0.7305 x-20066$ based on the values where $\left(x_{27677}, y_{27677}\right)$, $\left(x_{27678}, y_{27678}\right), \ldots,\left(x_{27705}, y_{27705}\right)$. Along formula (15), the next failure time could be estimated as follows:

$$
\begin{aligned}
& \text { The next failure time }=\frac{1}{0.7305}\left(\frac{13200}{\sqrt{3600}}+20066\right) \\
& \doteq 27756.33 \text { (hours) }
\end{aligned}
$$

The real failure time is 27776 . Since the time point when we estimated the next failure time is 27705 , the remaining life time is 51.33 (hours). Hence, we could also see that the estimated next failure time by regression model based approach is close to the real one.

4.4. Computational Experiments of Regression Model Based Approach. In order to evaluate the performance of regression model based approach, we have also carried out computational experiments. Table 4 shows the values of MAPEs when comparing the residual life time estimated from proposed regression model based approach to the real residual life time. According to this table, we could find the trend that regression model based approach has good performance as the residual life time becomes close to zero, compared to Markov model based approach. However, we could also see that the regression model based approach has the limitation in estimating the next failure time when the residual life time is relatively long since the linear regression model has the tendency in being highly affected by abnormal data.

\section{Hybrid Approach}

Throughout case study and computational experiments for two approaches, we could find that Markov model based approach has more better performance than regression model based approach when the remaining residual life time is relatively long, while regression model based approach has more better performance in the opposite case. The Markov model based approach could have stable performance compared to the regression model based approach although it seems to give not good performance as the residual life time becomes closed to the failure time. On the contrary, the linear regression method is straightforward and practical, and it gives us the relative good performance when the residual life time is closed to the failure time compared to the Markov model based approach. However, it is so sensitive on some abnormal data that it often overestimates or underestimates them. For this reason, in this study we suggest the hybrid approach that applies both Markov model based approach and regression model based approach into estimating the next failure time. In the hybrid approach, until a certain vibration level, Markov model based approach is applied, and then, after over the certain vibration level, the regression model based approach is used to estimate the next failure time. The below formula shows us how to apply both approaches depending on the level of vibration, in the hybrid approach.

$$
\text { The next failure time }= \begin{cases}\mathrm{EFT}_{1}, & \left(y_{i}<\omega\right) \\ \mathrm{EFT}_{2}, & \text { Otherwise }\end{cases}
$$

where $\mathrm{EFT}_{1}$ and $\mathrm{EFT}_{2}$ represent the estimated next failure time by the Markov model based approach and that by the regression model based approach, respectively, and $\omega$ indicates the vibration index.

In order to minimize the MAPE, it is necessary to find the best $\omega$. To this end, we have carried out the experiments with increasing $\omega$ from $3000 / \sqrt{\mathrm{RPM}}$ to $12600 / \sqrt{\mathrm{RPM}}$ by 
TABLE 3: Data and variables used in the regression model based approach.

\begin{tabular}{|c|c|c|c|c|}
\hline$x_{i}$ & $y_{i}$ & $\bar{y}_{i}$ & $v_{i}$ & $\prod_{i=m}^{m+r-1} v_{i}$ \\
\hline 27606 & 136.703 & & & \\
\hline 27607 & 139.811 & & & \\
\hline 27608 & 143.092 & & & \\
\hline 27609 & 146.458 & & & \\
\hline 27610 & 149.736 & & & \\
\hline 27611 & 153.115 & & & \\
\hline 27612 & 156.455 & & & \\
\hline 27613 & 159.753 & & & \\
\hline 27614 & 163.064 & & & \\
\hline 27615 & 166.381 & 151.457 & 1 & 1 \\
\hline 27616 & 169.561 & 154.742 & 1 & 1 \\
\hline 27617 & 172.655 & 158.027 & 1 & 1 \\
\hline 27618 & 175.649 & 161.283 & 1 & 1 \\
\hline 27619 & 178.551 & 164.492 & 1 & 1 \\
\hline 27620 & 181.348 & 167.653 & 1 & 1 \\
\hline 27621 & 183.963 & 170.738 & 1 & 1 \\
\hline 27622 & 186.444 & 173.737 & 1 & 1 \\
\hline 27623 & 188.752 & 176.637 & 1 & 1 \\
\hline 27624 & 190.983 & 179.429 & 1 & 1 \\
\hline 27625 & 193.030 & 182.094 & 1 & 1 \\
\hline 27626 & 194.859 & 184.623 & 1 & 1 \\
\hline 27627 & 196.451 & 187.003 & 1 & 1 \\
\hline 27628 & 197.835 & 189.222 & 1 & 1 \\
\hline 27629 & 198.999 & 191.266 & 1 & 1 \\
\hline 27630 & 199.979 & 193.129 & 1 & 1 \\
\hline 27631 & 200.711 & 194.804 & 1 & 1 \\
\hline 27632 & 201.280 & 196.288 & 1 & 1 \\
\hline 27633 & 201.615 & 197.574 & 1 & 1 \\
\hline 27634 & 201.790 & 198.655 & 1 & 0 \\
\hline 27635 & 201.793 & 199.531 & 1 & 0 \\
\hline 27636 & 201.542 & 200.199 & 1 & 0 \\
\hline 27637 & 201.101 & 200.664 & 1 & 0 \\
\hline 27638 & 200.427 & 200.924 & 0 & 0 \\
\hline 27639 & 199.639 & 200.988 & 0 & 0 \\
\hline 27640 & 198.696 & 200.859 & 0 & 0 \\
\hline 27641 & 197.652 & 200.553 & 0 & 0 \\
\hline 27642 & 196.376 & 200.063 & 0 & 0 \\
\hline 27643 & 194.990 & 199.400 & 0 & 0 \\
\hline 27644 & 193.359 & 198.557 & 0 & 0 \\
\hline 27645 & 191.658 & 197.544 & 0 & 0 \\
\hline 27646 & 189.901 & 196.380 & 0 & 0 \\
\hline 27647 & 187.956 & 195.065 & 0 & 0 \\
\hline 27648 & 186.044 & 193.627 & 0 & 0 \\
\hline 27649 & 184.097 & 192.073 & 0 & 0 \\
\hline 27650 & 181.980 & 190.401 & 0 & 0 \\
\hline 27651 & 179.913 & 188.627 & 0 & 0 \\
\hline 27652 & 177.806 & 186.770 & 0 & 0 \\
\hline 27653 & 175.629 & 184.834 & 0 & 0 \\
\hline 27654 & 173.501 & 182.849 & 0 & 0 \\
\hline 27655 & 171.427 & 180.825 & 0 & 0 \\
\hline
\end{tabular}

TABLE 3: Continued.

\begin{tabular}{|c|c|c|c|c|}
\hline$x_{i}$ & $y_{i}$ & $\bar{y}_{i}$ & $v_{i}$ & $\prod_{i=m}^{m+r-1} v_{i}$ \\
\hline 27656 & 169.394 & 178.775 & 0 & 0 \\
\hline 27657 & 167.483 & 176.727 & 0 & 0 \\
\hline 27658 & 165.574 & 174.680 & 0 & 0 \\
\hline 27659 & 163.767 & 172.647 & 0 & 0 \\
\hline 27660 & 162.018 & 170.651 & 0 & 0 \\
\hline 27661 & 160.327 & 168.693 & 0 & 0 \\
\hline 27662 & 158.764 & 166.788 & 0 & 0 \\
\hline 27663 & 157.409 & 164.966 & 0 & 0 \\
\hline 27664 & 156.072 & 163.223 & 0 & 0 \\
\hline 27665 & 154.883 & 161.569 & 0 & 0 \\
\hline 27666 & 153.795 & 160.009 & 0 & 0 \\
\hline 27667 & 152.810 & 158.542 & 0 & 0 \\
\hline 27668 & 151.905 & 157.175 & 0 & 0 \\
\hline 27669 & 151.295 & 155.928 & 0 & 0 \\
\hline 27670 & 150.824 & 154.808 & 0 & 0 \\
\hline 27671 & 150.363 & 153.812 & 0 & 0 \\
\hline 27672 & 150.066 & 152.942 & 0 & 0 \\
\hline 27673 & 149.992 & 152.200 & 0 & 0 \\
\hline 27674 & 149.918 & 151.585 & 0 & 0 \\
\hline 27675 & 150.113 & 151.108 & 0 & 0 \\
\hline 27676 & 150.297 & 150.758 & 0 & 0 \\
\hline 27677 & 150.689 & 150.546 & 1 & 1 \\
\hline 27678 & 151.243 & 150.480 & 1 & 1 \\
\hline 27679 & 151.726 & 150.523 & 1 & 1 \\
\hline 27680 & 152.399 & 150.680 & 1 & 1 \\
\hline 27681 & 153.234 & 150.968 & 1 & 1 \\
\hline 27682 & 154.061 & 151.367 & 1 & 1 \\
\hline 27683 & 154.907 & 151.859 & 1 & 1 \\
\hline 27684 & 155.768 & 152.444 & 1 & 1 \\
\hline 27685 & 156.715 & 153.104 & 1 & 1 \\
\hline 27686 & 157.694 & 153.843 & 1 & 1 \\
\hline 27687 & 158.639 & 154.638 & 1 & 1 \\
\hline 27688 & 159.699 & 155.484 & 1 & 1 \\
\hline 27689 & 160.672 & 156.379 & 1 & 1 \\
\hline 27690 & 161.683 & 157.307 & 1 & 1 \\
\hline 27691 & 162.687 & 158.252 & 1 & 1 \\
\hline 27692 & 163.702 & 159.217 & 1 & 1 \\
\hline 27693 & 164.565 & 160.182 & 1 & 1 \\
\hline 27694 & 165.367 & 161.142 & 1 & 1 \\
\hline 27695 & 166.084 & 162.079 & 1 & 1 \\
\hline 27696 & 166.677 & 162.977 & 1 & 1 \\
\hline 27697 & 167.268 & 163.840 & 1 & 1 \\
\hline 27698 & 167.799 & 164.650 & 1 & 1 \\
\hline 27699 & 168.114 & 165.395 & 1 & 1 \\
\hline 27700 & 168.363 & 166.063 & 1 & 1 \\
\hline 27701 & 168.493 & 166.643 & 1 & 1 \\
\hline 27702 & 168.592 & 167.132 & 1 & \\
\hline 27703 & 168.541 & 167.530 & 1 & \\
\hline 27704 & 168.372 & 167.830 & 1 & \\
\hline 27705 & 168.135 & 168.035 & 1 & \\
\hline
\end{tabular}


TABLE 4: Computational experiment results of regression model based approach.

\begin{tabular}{|c|c|c|c|c|c|c|c|c|}
\hline \multirow{2}{*}{$\begin{array}{l}\text { RLT }^{*} \\
\text { No. }^{\dagger}\end{array}$} & \multicolumn{2}{|c|}{10} & \multicolumn{2}{|c|}{15} & \multicolumn{2}{|c|}{20} & \multicolumn{2}{|c|}{25} \\
\hline & Est. $^{*}$ & MAPE & Est. & MAPE & Est. & MAPE & Est. & MAPE \\
\hline 1 & 9.323 & $7.01 \%$ & 18.692 & $21.91 \%$ & 29.264 & $37.61 \%$ & 45.582 & $58.32 \%$ \\
\hline 2 & 9.836 & $1.65 \%$ & 21.562 & $35.89 \%$ & 36.439 & $58.25 \%$ & 87.836 & $111.38 \%$ \\
\hline 3 & 11.632 & $15.09 \%$ & 24.609 & $48.52 \%$ & 38.227 & $62.61 \%$ & 58.742 & $80.59 \%$ \\
\hline 4 & 6.703 & $39.47 \%$ & 13.807 & $8.28 \%$ & 22.950 & $13.74 \%$ & 37.241 & $39.33 \%$ \\
\hline 5 & 4.897 & $68.52 \%$ & 10.003 & $39.97 \%$ & 17.013 & $16.14 \%$ & 26.157 & $4.52 \%$ \\
\hline 6 & 10.510 & $4.97 \%$ & 21.993 & $37.81 \%$ & 35.793 & $56.61 \%$ & 59.961 & $82.30 \%$ \\
\hline 7 & 9.318 & $7.06 \%$ & 18.093 & $18.69 \%$ & 27.484 & $31.52 \%$ & 40.181 & $46.58 \%$ \\
\hline 8 & 6.443 & $43.26 \%$ & 14.471 & $3.59 \%$ & 26.705 & $28.71 \%$ & 181.786 & $151.64 \%$ \\
\hline 9 & 10.946 & $9.03 \%$ & 22.764 & $41.12 \%$ & 36.031 & $57.22 \%$ & 56.923 & $77.93 \%$ \\
\hline 10 & 9.669 & $3.36 \%$ & 18.323 & $19.94 \%$ & 27.370 & $31.12 \%$ & 39.112 & $44.02 \%$ \\
\hline 11 & 9.845 & $1.57 \%$ & 20.124 & $29.17 \%$ & 31.880 & $45.80 \%$ & 50.038 & $66.73 \%$ \\
\hline 12 & 6.977 & $35.62 \%$ & 14.004 & $6.87 \%$ & 22.399 & $11.32 \%$ & 33.762 & $29.82 \%$ \\
\hline 13 & 7.267 & $31.65 \%$ & 14.912 & $0.59 \%$ & 23.800 & $17.35 \%$ & 36.211 & $36.63 \%$ \\
\hline Average & \multicolumn{2}{|c|}{$20.64 \%$} & \multicolumn{2}{|c|}{$24.03 \%$} & \multicolumn{2}{|c|}{$36.00 \%$} & \multicolumn{2}{|c|}{$63.83 \%$} \\
\hline Total ave & \multicolumn{8}{|c|}{$36.12 \%$} \\
\hline
\end{tabular}

${ }^{*}$ Residual life time (unit: hour).

${ }^{\dagger}$ Failure event number.

${ }^{\ddagger}$ Residual life time estimated by regression model based approach (unit: hour).

TABLE 5: Test results depending on $\omega$.

\begin{tabular}{lc}
\hline$\omega$ & Average MAPE \\
\hline $3000 / \sqrt{\mathrm{RPM}}$ & $48.64 \%$ \\
$3600 / \sqrt{\mathrm{RPM}}$ & $47.88 \%$ \\
$4200 / \sqrt{\mathrm{RPM}}$ & $47.04 \%$ \\
$4800 / \sqrt{\mathrm{RPM}}$ & $45.85 \%$ \\
$5400 / \sqrt{\mathrm{RPM}}$ & $43.93 \%$ \\
$6000 / \sqrt{\mathrm{RPM}}$ & $42.13 \%$ \\
$6600 / \sqrt{\mathrm{RPM}}$ & $40.05 \%$ \\
$7200 / \sqrt{\mathrm{RPM}}$ & $38.02 \%$ \\
$7800 / \sqrt{\mathrm{RPM}}$ & $37.25 \%$ \\
$8400 / \sqrt{\mathrm{RPM}}$ & $37.09 \%$ \\
$9000 / \sqrt{\mathrm{RPM}}$ & $37.39 \%$ \\
$9600 / \sqrt{\mathrm{RPM}}$ & $37.32 \%$ \\
$10200 / \sqrt{\mathrm{RPM}}$ & $37.85 \%$ \\
$10800 / \sqrt{\mathrm{RPM}}$ & $37.98 \%$ \\
$11400 / \sqrt{\mathrm{RPM}}$ & $38.27 \%$ \\
$12000 / \sqrt{\mathrm{RPM}}$ & $38.48 \%$ \\
$12600 / \sqrt{\mathrm{RPM}}$ & $38.69 \%$ \\
\hline
\end{tabular}

$600 / \sqrt{\text { RPM }}$. Table 5 shows the result of experiments depending on the changes of $\omega$. It shows us that $\omega=8400 / \sqrt{\mathrm{RPM}}$ has the best performance.

\section{Conclusion}

This study has dealt with the approaches for estimating the next failure time of offshore plant equipment, gas compressor. How to estimate the next failure time based on vibration data has been proposed by three approaches: regression model based approach, Markov model based approach, and their hybrid approach. In the Markov model based approach, based on the gathered vibration state-timestamp data, the next failure time of a gas compressor has been estimated by the finite state continuous time Markov model theory. In the regression model based approach, the linear regression model using moving average filter has been proposed. The hybrid approach takes the advantage of two approaches. Depending on the vibration level, one of two approach is applied to estimate the next failure time.

To show the usefulness of the proposed approaches, case examples and computational experiments based on shaft vibration sensor data were introduced in a case study. Although the proposed approaches have some limitations in fully evaluating the usability in the real field due to the limited examples used in the case study, we believe that it will provide offshore operation companies with a reference for doing the improved maintenance planning and decreasing equipment downtime due to unexpected failures.

We can think of several future research works. First, after suitably tuning the parameters (e.g., $n, \eta$, and regression parameters) used in our approaches based on lots of field data, our approaches could be applied to not only gas compressor but also other pieces of equipment, the failure of which could be being monitored by vibration signals. Second, the linear regression model could be improved to the more elaborate regression model by considering the trade-offs between generalizability and goodness of fit. In general case, since exponential model seems to be more suitable for the degradation model of mechanical system, it is valuable to consider more complex regression model rather than the first-order linear regression model. Third, it is possible to 
specify the approaches in detail considering the segmentation of ocean environment and equipment operating mission profile. Fourth, in this study, based on engineers interview, we assumed that the state transition in the Markov model is time independent. However, as the future research issue, it is meaningful to consider the time dependency of deterioration process of compressor. In addition, we did not consider realtime interactive update of the parameters used in the Markov model. However, since the degradation process is changing over time due to various uncertainties, it is also valuable to consider the real-time update by using the methods such as MCMC (Monte Carlo Markov Chain) and classic control models. Finally, other probabilistic methods such as Bayesian network and artificial neural network could be applied to develop a more elaborated prognostic algorithm.

\section{Competing Interests}

The authors declare that there is no conflict of interests regarding the publication of this paper.

\section{Acknowledgments}

This research was financially supported by Korea Evaluation Institute of Industrial Technology (KEIT, Republic of Korea) and the Ministry of Trade, Industry and Energy (MOTIE, Republic of Korea) through the core technology development program of Industrial Convergence Technology (10045212, predictive maintenance system for the integrated and intelligent operation of offshore plant).

\section{References}

[1] Y. Peng, M. Dong, and M. J. Zuo, "Current status of machine prognostics in condition-based maintenance: a review," International Journal of Advanced Manufacturing Technology, vol. 50, no. 1-4, pp. 297-313, 2010.

[2] S. Telford, M. Mazhar, and I. Howard, "Condition based maintenance (CBM) in the oil and gas industry: an overview of methods and techniques," in Proceedings of the International Conference on Industrial Engineering and Operations Management, pp. 1152-1159, Kuala Lumpur, Malaysia, January 2011.

[3] A. Prajapati, J. Bechtel, and S. Ganesan, "Condition based maintenance: a survey," Journal of Quality in Maintenance Engineering, vol. 18, no. 4, pp. 384-400, 2012.

[4] J. Zhu, Y. Li, W. Wang et al., "Offshore adaptability of the $\mathrm{CO}_{2}$ pre-cooling dual nitrogen expander natural gas liquefaction process," Advanced Materials Research, vol. 608-609, pp. 13691374, 2013.

[5] M. Bevilacqua and M. Braglia, "The analytic hierarchy process applied to maintenance strategy selection," Reliability Engineering and System Safety, vol. 70, no. 1, pp. 71-83, 2000.

[6] R. Kothamasu, S. H. Huang, and W. H. VerDuin, "System health monitoring and prognostics-a review of current paradigms and practices," International Journal of Advanced Manufacturing Technology, vol. 28, no. 9, pp. 1012-1024, 2006.

[7] D. T. Griffith, N. C. Yoder, B. Resor, J. White, and J. Paquette, "Structural health and prognostics management for the enhancement of offshore wind turbine operations and maintenance strategies," Wind Energy, vol. 17, no. 11, pp. 1737-1751, 2014.
[8] J. Lee, "Teleservice engineering in manufacturing: challenges and opportunities," International Journal of Machine Tools \& Manufacture, vol. 38, no. 8, pp. 901-910, 1998.

[9] L. D. Lee, "Using wireless technology and the internet for predictive maintenance," Hydrocarbon Processing, vol. 80, no. 5, pp. 77-80, 2001.

[10] L. Dieulle, C. Berenguer, A. Grall, and M. Roussignol, "Continuous time predictive maintenance scheduling for a deteriorating system," in Proceedings of the IEEE Annual Symposium on Reliability and Maintainability, pp. 150-155, Philadelphia, Pa, USA, January 2001.

[11] A. Grall, C. Bérenguer, and L. Dieulle, "A condition-based maintenance policy for stochastically deteriorating systems," Reliability Engineering and System Safety, vol. 76, no. 2, pp. 167-180, 2002.

[12] C.-C. Lin and H.-Y. Tseng, "A neural network application for reliability modelling and condition-based predictive maintenance," International Journal of Advanced Manufacturing Technology, vol. 25, no. 1-2, pp. 174-179, 2005.

[13] W. J. Moore and A. G. Starr, "An intelligent maintenance system for continuous cost-based prioritisation of maintenance activities," Computers in Industry, vol. 57, no. 6, pp. 595-606, 2006.

[14] S.-J. Wu, N. Gebraeel, M. A. Lawley, and Y. Yih, "A neural network integrated decision support system for condition-based optimal predictive maintenance policy," IEEE Transactions on Systems, Man, and Cybernetics Part A:Systems and Humans, vol. 37, no. 2, pp. 226-236, 2007.

[15] W. Wu, J. Hu, and J. Zhang, "Prognostics of machine health condition using an improved ARIMA-based prediction method," in Proceedings of the 2nd IEEE Conference on Industrial Electronics and Applications (ICIEA '07), pp. 1062-1067, IEEE, Harbin, China, May 2007.

[16] H. M. Hashemian and W. C. Bean, "State-of-the-art predictive maintenance techniques," IEEE Transactions on Instrumentation and Measurement, vol. 60, no. 10, pp. 3480-3492, 2011.

[17] A. Gruber, S. Yanovski, and I. Ben-Gal, "Condition-based maintenance via simulation and A targeted bayesian network metamodel," Quality Engineering, vol. 25, no. 4, pp. 370-384, 2013.

[18] J. Lee, F. Wu, W. Zhao, M. Ghaffari, L. Liao, and D. Siegel, “Prognostics and health management design for rotary machinery systems-reviews, methodology and applications," Mechanical Systems and Signal Processing, vol. 42, no. 1-2, pp. 314-334, 2014.

[19] C. Bunks, D. McCarthy, and T. Al-Ani, "Condition-based maintenance of machines using hidden Markov models," Mechanical Systems and Signal Processing, vol. 14, no. 4, pp. 597-612, 2000.

[20] S. Ambani, L. Li, and J. Ni, "Condition-based maintenance decision-making for multiple machine systems," Journal of Manufacturing Science and Engineering, Transactions of the ASME, vol. 131, no. 3, pp. 0310091-0310099, 2009.

[21] X.-S. Si, W. Wang, C.-H. Hu, and D.-H. Zhou, "Remaining useful life estimation-a review on the statistical data driven approaches," European Journal of Operational Research, vol. 213, no. 1, pp. 1-14, 2011.

[22] W. Wang and H. B. A. Majid, "Reliability data analysis and modelling of offshore oil platform plant," Journal of Quality in Maintenance Engineering, vol. 6, no. 4, pp. 287-295, 2000.

[23] N. Arthur and M. Dunn, "Effective condition-based maintenance of reciprocating compressors on an offshore oil and gas installation," in Proceedings of International Conference on Compressors and Their Systems, pp. 213-221, London, UK, 2001. 
[24] P. Caselitz and J. Giebhardt, "Advanced maintenance and repair for offshore wind farms using fault prediction techniques," in Proceedings of the World Wind Energy Conference, Berlin, Germany, 2002.

[25] P. K. Dey, S. O. Ogunlana, and S. Naksuksakul, "Risk-based maintenance model for offshore oil and gas pipelines: a case study," Journal of Quality in Maintenance Engineering, vol. 10, no. 3, pp. 169-183, 2004.

[26] A. G. Eleye-Datubo, A. Wall, A. Saajedi, and J. Wang, "Enabling a powerful marine and offshore decision-support solution through bayesian network technique," Risk Analysis, vol. 26, no. 3, pp. 695-721, 2006.

[27] A. G. Eleye-Datubo, A. Wall, and J. Wang, "Marine and offshore safety assessment by incorporative risk modeling in a fuzzyBayesian network of an induced mass assignment paradigm," Risk Analysis, vol. 28, no. 1, pp. 95-112, 2008.

[28] E. Migueláñez and D. Lane, "Predictive diagnosis for offshore wind turbines using holistic condition monitoring," in Proceedings of the IEEE OCEANS, pp. 1-7, Seattle, Wash, USA, September 2010.

[29] H. Hussin, M. Muhammad, F. M. Hashim, and S. N. Ibrahim, "A practical method for analyzing offshore gas compressor system maintenance data," AIP Conference Proceedings, vol. 1285, no. 1, pp. 207-221, 2010.

[30] A. H. de Andrade Melani, D. W. R. Silva, and G. F. M. Souza, "Use of Bayesian network to support risk-based analysis of LNG carrier loading operation," in Proceedings of the Probabilistic Safety Assessment and Management (PSAM '14), Honolulu, Hawaii, USA, June 2014.

[31] S. Cho, H. Jun, J. Shin, and S. Choi, "A study on estimating the next failure time of lng fpso compressor," Korean Journal of Computational Design and Engineering, vol. 19, no. 3, pp. 203213, 2014.

[32] S. Cho, H.-B. Jun, J.-H. Shin, H.-J. Hwang, and C. Ha, "A study on the development of prognosis system for offshore plant equipment," in Proceedings of the 25th International Ocean and Polar Engineering Conference (ISOPE '15), pp. 464-470, Kona, Hawaii, USA, June 2015.

[33] B. Jones, I. Jenkinson, Z. Yang, and J. Wang, "The use of Bayesian network modelling for maintenance planning in a manufacturing industry," Reliability Engineering and System Safety, vol. 95, no. 3, pp. 267-277, 2010.

[34] SINTEF, Offshore reliability data, OREDA participants, 2009.

[35] R. K. Mobley, An Introduction to Predictive Maintenance, Elsevier, 2002.

[36] P. W. Tse and D. P. Atherton, "Prediction of machine deterioration using vibration based fault trends and recurrent neural networks," Journal of Vibration and Acoustics, vol. 121, no. 3, pp. 355-362, 1999.

[37] D. Anderson, "Introduction to stochastic processes with applications in the biosciences," Tech. Rep., University of Wisconsin at Madison, 2013.

[38] P. Goodwin and R. Lawton, "On the asymmetry of the symmetric MAPE," International Journal of Forecasting, vol. 15, no. 4, pp. 405-408, 1999. 


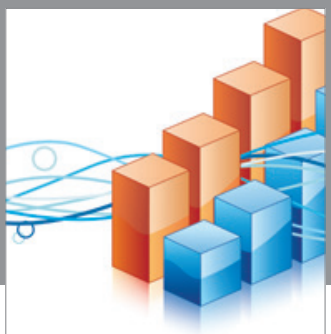

Advances in

Operations Research

vatem alat4

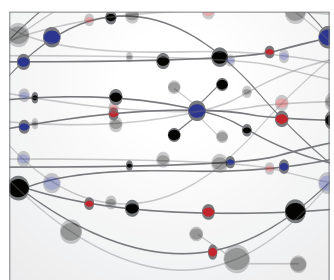

\section{The Scientific} World Journal
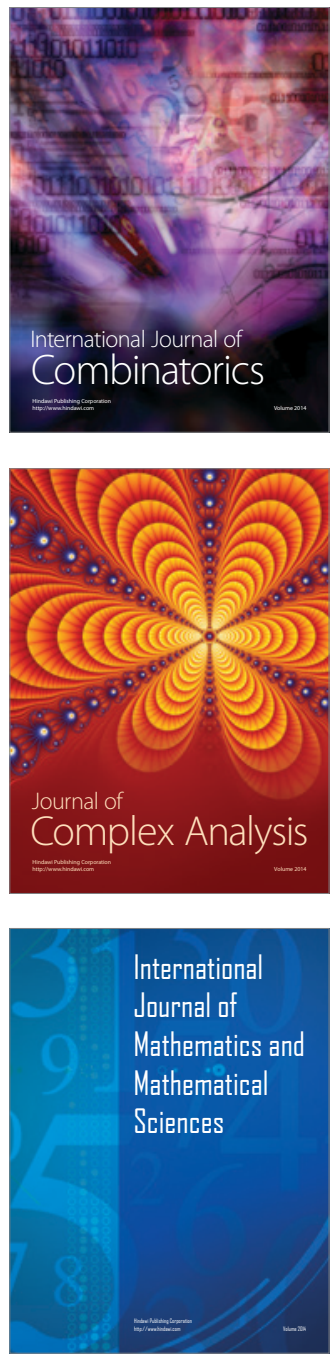
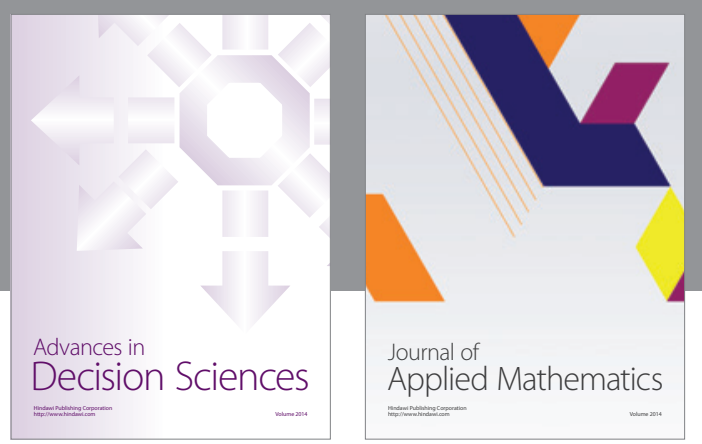

Algebra

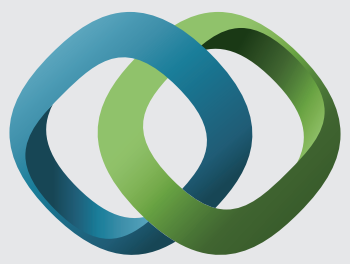

\section{Hindawi}

Submit your manuscripts at

http://www.hindawi.com
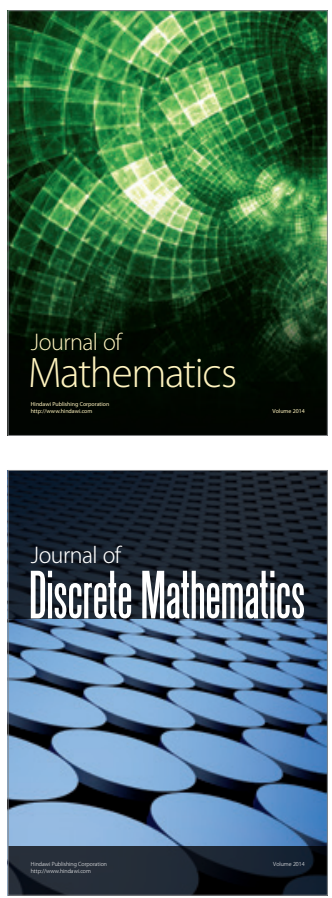

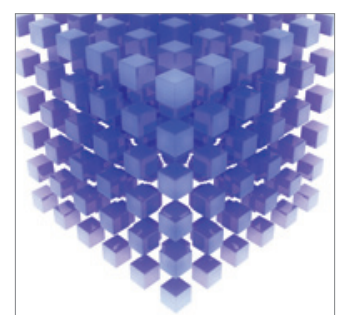

Mathematical Problems in Engineering
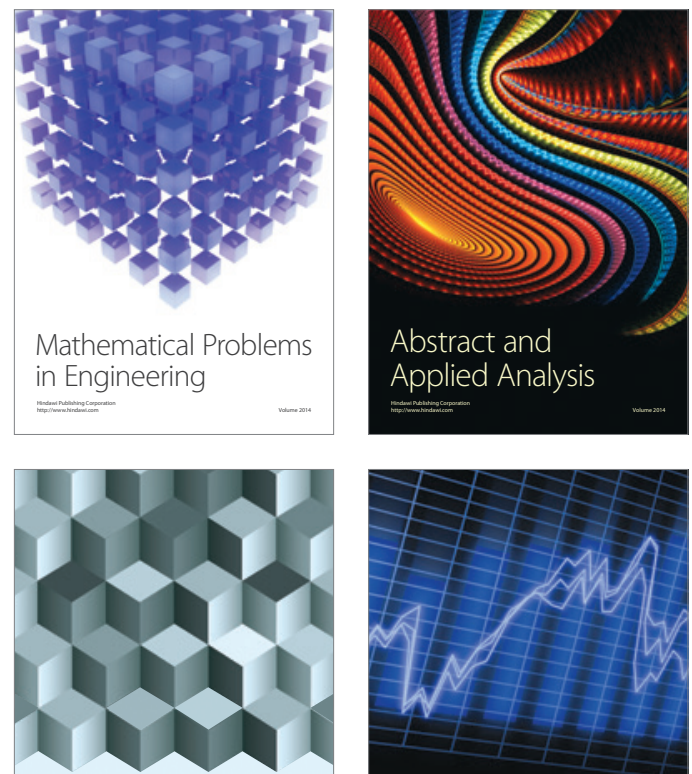

Journal of

Function Spaces

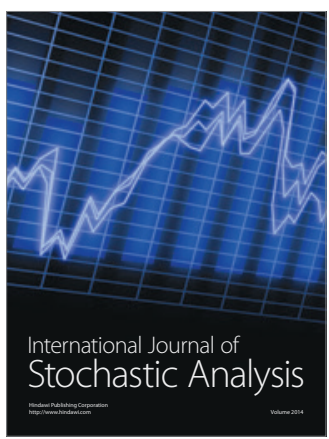

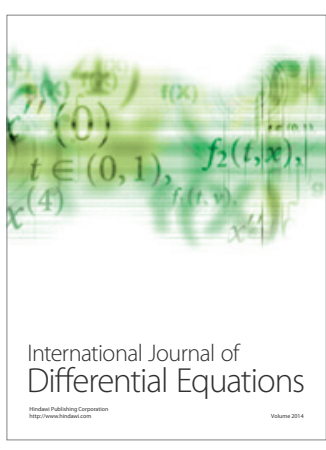
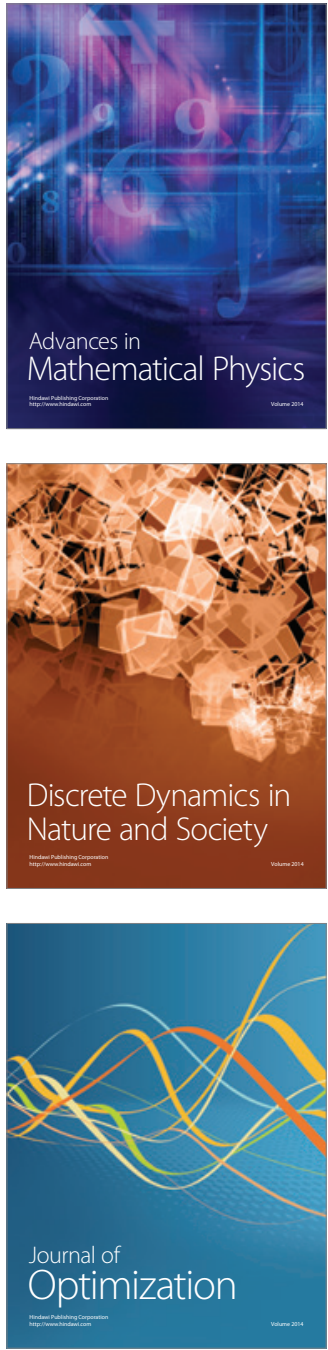